\title{
Das „Reichsvolksschulgesetz" und seine Auswirkungen auf die Mädchenbildung. Ein Schritt zur Gleichstellung der Geschlechter in österreichischen Grundschulen?
}

\author{
Nina Hechenblaikner \\ Kerngebiet: Österreichische Geschichte \\ eingereicht bei: Ass.-Prof. i. R. Mag. Dr. Hermann Kuprian \\ eingereicht im: SoSe 2016 \\ Rubrik: SE-Arbeit
}

\begin{abstract}
The "Reichsvolksschulgesetz" and its effects on the education of girls. A step towards the equalisation of genders in Austrian primary schools?

The following paper deals with the question of how the "Reichsvolksschulgesetz" influenced and changed the Austrian primary school system. Therefore, parts of the law are analysed regarding their purpose and how they were implemented. This paper also examines the influences that this law had on educational opportunities for girls. It will be shown that an equality of genders was not the purpose of the "Reichsvolksschulgesetz".
\end{abstract}

\section{Einleitung}

"Der Mensch ist, was [...] er als Mensch sein soll, erst durch Bildung."

Dass Bildung ein wichtiges Gut darstellt, ist heute sicherlich unumstritten. Der Besuch einer Volksschule ist ein Grundstein unserer Bildung und für die meisten in Österreich lebenden Menschen Normalität. Der Weg bis zu dieser Selbstverständlichkeit des Schulbesuches war jedoch lang. Ein wichtiger Schritt in der Entwicklung des österreichischen Schulwesens war das Gesetz vom 14. Mai 1869, „RGBI. Nr. 62“, durch welches die Grundsätze des Unterrichtswesens bezüglich der Volksschulen festgelegt wurden.

1 Georg Wilhelm Friedrich Hegel, Phänomenologie des Geistes, Stuttgart 1999, S. 295. 
Diese Arbeit beschäftigt sich mit der Frage, wie dieses „Reichsvolksschulgesetz" die Grundschule im Gebiet des heutigen Österreich veränderte. Anhand von Analysen des Gesetzes selbst sowie weiteren Schlüsseldokumenten werden die Reformen genauer unter die Lupe genommen. Sowohl die kausalen Ursachen und Befürworter_innen der Neuerung als auch der erbitterte Widerstand von diversen Seiten werden angeführt. Es wird erörtert, ob das "Reichsvolksschulgesetz" als universale Verbesserung des Bildungswesens gesehen werden konnte. In einem weiteren Teil wird der Frage nachgegangen, welche Veränderungen sich in Sachen Bildung für Mädchen ergaben. Es gilt zu analysieren, inwiefern das "Reichsvolksschulgesetz" die Chancen für junge Frauen beeinflusste oder verbesserte. Ein zentraler Punkt dafür wird die Frage sein, ob es durch das neue Gesetz zu einer Gleichstellung der Geschlechter in den Elementarschulen kam, beziehungsweise ob eine Gleichstellung überhaupt das Ziel dieser Reform war.

Sowohl die Entwicklung des österreichischen Volksschulwesens als auch die Mädchenbildung wurden in der Fachliteratur bereits aufgearbeitet. Helmut Engelbrechts fünfbändige "Geschichte des österreichischen Bildungswesens"2 kann als Standardwerk zu dieser Thematik gesehen werden. Auch Ludwig Boyers „Elementarschulen und Elementarunterricht in Österreich" “3 ist hier zu nennen. Für den Bereich der Mädchenbildung ist

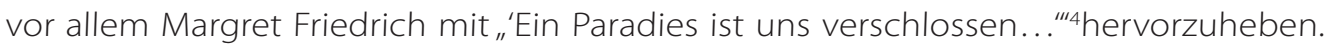
Die These, die dieser Arbeit zugrunde liegt, lautet, dass das "Reichsvolksschulgesetz" zwar eine wichtige Innovation für das österreichische Grundschulwesen war, die Bildungschancen für Mädchen sich dadurch allerdings nur unwesentlich verbesserten.

\section{Das österreichische Grundschulwesen vor dem „Reichsvolksschulgesetz"}

1774 wurde unter Maria Theresia die „Allgemeine Schulordnung für die deutschen Normal-, Haupt- und Trivialschulen in sämmtlichen Kayserl. Königl. Erbländern“ eingeführt. Die Bestrebung dahinter war das Wohl der Länder. Im Vorwort zu diesem Gesetz gibt Maria Theresia an, dass die „Erziehung der Jugend, beyderley Geschlechtes, als die wichtigste Grundlage der wahren Glückseligkeit“5 zu sehen sei. Das Gesetz regelte die Schulpflicht, -organisation und -aufsicht. Für das Grundschulwesen waren drei Schularten vorgesehen. Ein- bis zweiklassige Trivialschulen sollten an jedem Pfarrort eingerichtet werden, dreiklassige Hauptschulen in jeder größeren Stadt, vierklassige Normalschulen nur in Provinzhauptstädten. Sie dienten auch der Ausbildung von Lehrkräften. ${ }^{6}$

2 Für diese Arbeit hervorzuheben ist: Helmut Engelbrecht, Geschichte des österreichischen Bildungswesens. Erziehung und Unterricht auf dem Boden Österreichs. Von 1848 bis zum Ende der Monarchie, Bd. 4, Wien 1986.

3 Ludwig Boyer, Elementarschulen und Elementarunterricht in Österreich. Illustrierte Chronik der Schul- und Methodengeschichte von den ältesten Quellen bis zum Ende des 19. Jahrhunderts, Graz 2012.

4 Margret Friedrich, „Ein Paradies ist uns verschlossen...." Zur Geschichte der schulischen Mädchenerziehung in Österreich im „langen“ 19. Jahrhundert, Wien-Köln-Weimar 1999.

5 Allgemeine Schulordnung für die deutschen Normal- Haupt- und Trivialschulen in sämmtlichen kaiserl. Königl. Erbländern i. d. F. 6. Dezember 1774, S. 4, [https://www.digital.wienbibliothek.at/wbrobv/content/ titleinfo/1843007], eingesehen 14.3.2018.

$6 \quad$ Helmut Engelbrecht, Geschichte des österreichischen Bildungswesens. Erziehung und Unterricht auf dem Boden Österreichs. Von der frühen Aufklärung bis zur Revolution, Bd. 3, Wien 1984, S. 103-104. 
Vor dem "Reichsvolksschulgesetz" wurde das österreichische Grundschulwesen durch die "Politische Verfassung der deutschen Schule in den k. auch k. u. k. deutschen Erbstaaten"7 geregelt, die 1805 verabschiedet wurde und 1806 in Kraft trat. Da die Aufsicht über die Schulen noch der Kirche oblag, war diese Verfassung stark von deren Einfluss geprägt. Die Volksschule, die damals noch "Trivialschule“8 genannt wurde, war für Kinder aus bäuerlichen Verhältnissen gedacht und sollte ihnen nur das Nötigste beibringen.

"So ist in Trivialschulen dahin zu arbeiten, daß darin den Kindern die geoffenbarte Religion Jesu Christi gut und herzeindringlich gelehrt werde, und daß sie über die Dinge, mit welchen sie umgehen, und über die Verhältnisse, in denen sie sich befinden [...] die richtigen Anweisungen bekommen, um die Dinge und Verhältnisse so zu benutzen, wie es die christliche Sittenlehre vorschreibt."

Auf dem Lehrplan standen außer Religionsunterricht nur Lesen, Schreiben und Rechnen sowie "eine praktische Anweisung einige Aufsätze zu machen.."10 Alles andere, wie etwa das Wissen, das zum Übertritt in das Gymnasium nötig war, war entbehrlich und durfte nicht unterrichtet werden. Die Hauptaufgabe der Volksschule zu dieser Zeit war, den Kindern christliche Normen und Gehorsam gegenüber Obrigkeiten beizubringen. Die Schulen „sollten vor allem zur Pflichterfüllung, Sittlichkeit und Religiosität erziehen."11

1848 kam die liberale Regierung zu dem Schluss, dass sie ihre „Forderungen nach Verwirklichung der Glaubens-, Rede- und Pressefreiheit, nach Achtung der Menschenrechte, Verfassung und Volksvertretung"12 leichter erreichen könnte, wenn ein Großteil der Bevölkerung gebildet wäre. Um dies zu erreichen, wurde das "Ministerium für Cultus und Unterricht" eingeführt. In einem ersten Schritt kam es noch im selben Jahr zu einer pädagogischen Reform, da erkannt worden war, dass die Volksschule der Grundstein des Bildungssystems war. Das Ziel der Volksschule sollte es nun sein, Kindern die Fähigkeiten und das Wissen zu vermitteln, das sie benötigten, um später einen Beruf und ihre Rechte und Pflichten als österreichische Staatsbürger_innen auszuüben. Jedoch konnten nur kleine Teile der gewünschten Reform umgesetzt werden und so wurden die Rufe nach einer gesamten Reformierung des österreichischen Grundschulwesens immer lauter. ${ }^{13}$

7 O. A., Politische Verfassung der deutschen Schule in der k. auch k.k. deutschen Erbstaaten, Wien 1806, S. 15, [https://books.google.at/books?id=IB9OAAAAcAAJ\&amp;printsec=frontcover\&amp; hl=de\#v=onepage\&amp;q\& amp;f=false], eingesehen 14.3.2018.

8 Ebd., S. 15

9 Ebd.

10 Ebd.

11 Engelbrecht, Geschichte des österreichischen Bildungswesens, Bd. 3, S. 228.

12 MargretFriedrich, HatteVater Staat nurStieftöchter? Die Maßnahmen des österreichischen Unterrichtsministeriums zur Mädchenbildung 1848-1919, in: Brigitte Mazohl (Hrsg.), Bürgerliche Frauenkultur im 19. Jahrhundert (L'homme Schriften 2), Wien 1995, S. 301-342, hier S. 303.

13 Friedrich, Hatte Vater Staat nur Stieftöchter?, S. 303; Engelbrecht, Geschichte 4, S. 107-108. 


\section{Das „Reichsvolksschulgesetz"}

Das „Reichsvolksschulgesetz" war ein großer und wichtiger Schritt in der Reformierung des gesamten österreichischen Schulwesens. Es veränderte sowohl die Organisation als auch den Lehrauftrag der Grundschulen entscheidend und umfasste zahlreiche Neuerungen, die sowohl den Unterricht und die Organisation der Schulen als auch die Ausbildung der Lehrkräfte betrafen. Bis auf einige Abänderungen und Novellierungen blieb das „Reichsvolksschulgesetz" bis zur Einführung des „Schulgesetzwerkes" 1962 also fast 100 Jahre lang - in Kraft. ${ }^{14}$

Erstmals wurden bildungspolitische Forderungen von liberalen Gruppierungen, v. a. den Deutschliberalen, 1848 gestellt. Diese hatten schon früh den Wert von Bildung erkannt. Der endgültige Auslöser dafür, dass die Ansinnen der Liberalen ernst genommen wurden und eine gründliche Reformierung des österreichischen Grundschulwesens durchgeführt wurde, war die Niederlage der österreichischen Armee bei der Schlacht von Königgrätz 1866. Als nach der Schuld für das Unterliegen gesucht wurde, geriet das Schulwesen beziehungsweise die fehlende Bildung der Rekruten schnell in den Blickpunkt. Die Statistiken der Musterung von 1865 besagten, dass zwei Drittel der österreichischen Rekruten Analphabeten waren. ${ }^{15}$ Der Ausspruch „[b]ei Königgrätz hat der deutsche Schulmeister den österreichischen geschlagen" entwickelte sich nach dieser Einsicht zu einer geläufigen Beschreibung der Ereignisse. ${ }^{16}$ Als erste Maßnahme wurde daraufhin 1867 das 1860 aufgelöste "Ministerium für Cultus und Unterricht" wieder eingeführt. Der neue Unterrichtsminister Leopold Hasner von Artha veranlasste, dass Abgeordnete ins Ausland geschickt wurden, um die dortige Organisation des Volksschulwesens zu beobachten und zu beurteilen. Die Analyse dieser Recherchen kam zu dem Ergebnis, dass der Zustand des österreichischen Grundschulsystems sehr schlecht war. Beispielsweise waren die Lehrpläne veraltet und entsprachen nicht mehr den Normen der Zeit. Außerdem wurde festgestellt, dass es in Österreich nicht genügend Volksschulen gab, um alle schulpflichtigen Kinder zu unterrichten. ${ }^{17}$ Unterrichtsminister Hasner beschrieb die Situation des österreichischen Grundschulwesens in seinem "Motivenbericht" zur Gesetzesvorlage des "Reichsvolksschulgesetzes" folgendermaßen:

"Die Zahl der Schulen ist eine zu geringe, um die gesamte Schuljugend zu erfassen. Die Normen über die Schulpflicht werden nicht streng genug gehandhabt und die Zahl der Schulversäumnisse ist in einigen Gegenden eine beträchtliche; der Lehrplan der Volksschule entspricht nicht mehr ganz den Anforderungen und Bedürfnissen der Gegenwart, indem eine Anzahl von Lehrfächern, welche in keiner Volksschule fehlen dürfen, keine Aufnahme gefunden hat; die Lehrerbildung ist eine mangelhafte, die materielle Stellung

Boyer, Elementarschulen, S. 272

Engelbrecht, Geschichte 4, S. 16 und S. 112.

Boyer, Elementarschulen, S. 262.

Ebd., S. 263 und S. 272 
der Lehrer ist eine höchst ungünstige, für die Bildung der weiblichen Schuljugend ist in einer nicht genügenden Weise Sorge getragen."18

Eine wichtige Maßnahme zur Reformierung des Volksschulwesens war das "Gesetz vom 25. Mai 1868, wodurch grundsätzliche Bestimmungen über das Verhältniß der Schule zur Kirche erlassen“ wurden, kurz auch "Schule-Kirche-Gesetz" genannt. Es war ein erster Schritt zur Trennung von Kirche und Schule und besagte, dass die oberste Leitung der Schule dem Staat oblag, der Religionsunterricht hingegen unter dem Vorstand der Kirche stehe. ${ }^{19}$ Einige Paragraphen dieses Gesetzes finden sich, ganz oder teilweise, dann auch im „Reichsvolksschulgesetz" wieder.

\subsection{Bestimmungen}

Das "Reichsvolksschulgesetz" beinhaltete zahlreiche Bestimmungen, die sich mit den unterschiedlichsten Bereichen des Grundschulsystems befassten. Die insgesamt 78 Paragraphen waren in die Teilbereiche „allgemeine Volksschule, Bürgerschule, Schulbesuch, Lehrerbildung und Befähigung zum Lehramte, Fortbildung der Lehrer, Rechtsverhältnisse der Lehrer, Errichtung der Schulen, Aufwand des Volksschulwesens und Bestreitung desselben, Privatschulen und Schlussbestimmungen" gegliedert. 16 Paragraphen befassten sich mit den neuen Bestimmungen zur Volksschule. Dass sich im Verhältnis dazu nur wenige Paragraphen ${ }^{20}$ mit der Bürgerschule beschäftigten, liegt daran, dass nach $\$ 19$ zehn Paragraphen aus dem Bereich „allgemeine Volksschule“ übernommen wurden.

Die Aufgabe der Volksschule wurde durch das "Reichsvolksschulgesetz" neu definiert und lautete nun,

„die Kinder sittlich-religiös zu erziehen, deren Geistestätigkeiten zu entwickeln, sie mit den zur weiteren Ausbildung für das Leben erforderlichen Kenntnissen und Fertigkeiten auszustatten und die Grundlage für Heranbildung tüchtiger Menschen und Mitglieder des Gemeinwesens zu schaffen." ${ }^{21}$

Über die Formulierung „sittlich-religiös“ wurde heftig debattiert, zeigte sie doch klar, dass von nun an die religiöse Erziehung der Kinder endgültig nicht mehr das primäre Ziel der Volksschule war. Schulen, die ganz oder auch nur teilweise vom Staat erhalten wurden, mussten außerdem von nun an für alle österreichischen Staatsbürger_innen, unabhängig von ihrem Religionsbekenntnis, frei zugänglich sein.

18 Otto Glöckel, Geleitwort, in: Deutschösterreichisches Unterrichtsamt (Hrsg.), Zur Fünfzigjahrfeier des Reichsvolksschulgesetzes. Der Motivenbericht Hasners und die parlamentarischen Kommissionsberichte, Wien 1919, S. 11, zit. in Ludwig Boyer, Elementarschulen und Elementarunterricht in Österreich. Illustrierte Chronik der Schul- und Methodengeschichte von den ältesten Quellen bis zum Ende des 19. Jahrhunderts, Graz 2012, S. 272.

19 Gemäß §1 und 2 Gesetz wodurch grundsätzliche Bestimmungen über das Verhältniß der Schule zur Kirche erlassen werden i. d. F. 25. Mai 1868, RGBI Nr. 48, [http://alex.onb.ac.at/cgi-content/alex?aid=rgb\&datum=1868\&s ize $=45$ \&page $=125]$, eingesehen 9.3.2018.

20 Im Teilbereich „Bürgerschule” sind nur vier Paragraphen zu finden.

21 Gemäß §1 Reichsvolksschulgesetz i. d. F. 14. Mai 1869, RGBI. Nr. 62, [http://alex.onb.ac.at/cgi-content/alex?aid=rg b\&datum $=18690004 \&$ seite $=00000277$, eingesehen 9.3.2018. 
Es wurde zwar noch immer darauf geachtet, den Volksschüler_innen nicht zu viel Wissen zu vermitteln, dennoch wurde der Lehrplan erheblich erweitert. Während zuvor in Volksschulen oft nur Religion, Lesen, Schreiben und Rechnen unterrichtet wurden, sollte der Unterricht von nun an in folgenden Fächern erteilt werden: „Religion, Sprache, Rechnen, das Wissenswerteste aus Naturkunde, Erdkunde und Geschichte mit besonderer Rücksichtnahme auf das Vaterland und dessen Verfassung, Schreiben, Geometrische Formenlehre, Gesang, Leibesübungen." ${ }^{22}$

Mädchen waren zusätzlich „auch noch in weiblichen Handarbeiten und in der Haushaltskunde zu unterweisen." ${ }^{23}$ Es stand den Schulen frei, Schüler_innen auch in anderen Fächern zu unterrichten, wenn genügend Lehrkräfte vorhanden waren.

Die Stundenanzahl der einzelnen Fächer wurde durch das "Reichsvolksschulgesetz" noch nicht festgelegt. Stattdessen wurden die Lehrenden an den Lehrerbildungsanstalten durch die Verordnung des Ministeriums vom 12. Juli 1869, womit "Uebergangsbestimmungen" zur Durchführung des Volksschulgesetzes erlassen wurden, damit beauftragt, Lehrpläne zu erstellen und Lehrziele festzulegen. Im Anhang der Verordnung fanden sich Lehrpläne für Volksschulen, die der Orientierung dienen sollten und als erste wirkliche Lehrpläne gesehen werden können. Neben einer Stundentafel für die einzelnen Schulformen enthielten sie auch Hinweise zur Einteilung der Klassen in Abteilungen und zum Wechsel von unmittelbarem Unterricht durch die Lehrperson und stiller Beschäftigung, während sich die Lehrkraft einer anderen Abteilung widmete. Die von den Lehrerbildungsanstalten ausgearbeiteten Lehrpläne sollten der Bezirksschulbehörde vorgelegt werden und ab dem Schuljahr 1869/70 zur Anwendung kommen. ${ }^{24}$

Um den Lehrpersonen den Übergang zu erleichtern, mussten die Unterrichtsgegenstände, die durch das "Reichsvolksschulgesetz" neu in den Lehrplan aufgenommen worden waren, im Schuljahr 1869/70 erst ab der fünften Schulstufe als eigenständige Fächer unterrichtet werden. In den unteren Klassen sollte man die Inhalte dieser Unterrichtsgegenstände nach Möglichkeit „mit dem Anschauungs- und Leseunterricht in Verbindung [...] bringen.“25 Lehrkräfte, die nicht über die nötigen Kenntnisse verfügten, um die neuen Fächer zu unterrichten, waren dazu aufgefordert, „durch Privatfleiß die Lücke ihres Wissens auszufüllen, um sich zum Unterricht in den neu aufgenommenen Lehrgegenständen zu befähigen." ${ }^{26}$ Hierfür wurden Fortbildungskurse an den Lehrerbildungsanstalten angeboten.

Nach „\$16 der Übergangsbestimmungen zur Durchführung des Volksschulgesetzes” sollten bisherige Bezeichnungen wie etwa "Trivialschule" "Hauptschule" und "Normalhauptschule" entfallen. Von nun an sollten die Schulkategorien durch die Anzahl der Klassen, die an einer Schule bestanden, unterschieden und auch nach dieser benannt

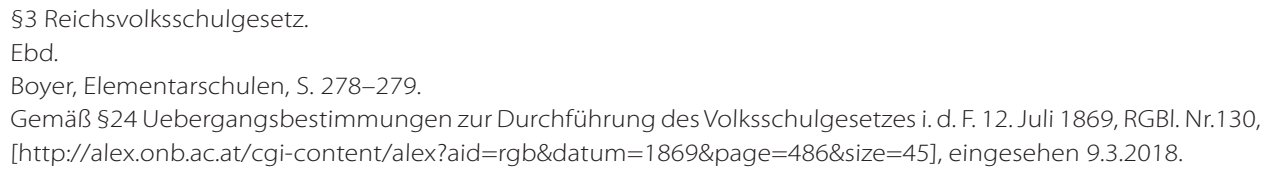


werden. Dabei galt, dass „jede Schülerzahl, für welche eine eigene Lehrkraft bestellt ist, [...] eine Classe [bildet]."27 Die Anzahl der Lehrenden wiederum richtete sich nach der Anzahl der Schüler_innen, die die Schule besuchten.

„Erreicht die Schülerzahl in drei aufeinander folgenden Jahren im Durschnitte 80, so muß unbedingt für eine zweite Lehrkraft, und steigt diese Zahl auf 160, für eine dritte gesorgt und nach diesem Verhältnisse die Zahl der Lehrer noch weiter vermehrt werden." ${ }^{\prime 2}$

Im Vergleich dazu sollte eine Lehrperson nach der politischen Verfassung der deutschen Schule von 1806, die bis dahin gegolten hatte, achtzig Schüler_innen betreuen; erst ab hundert Schüler_innen sollte ein zweites Klassenzimmer eingerichtet sowie eine zweite Lehrperson eingestellt werden. Die Landesschulbehörde behielt sich laut dem „Reichsvolksschulgesetz" außerdem vor, die maximale Anzahl von Schüler_innen, die eine Lehrkraft betreuen konnte, herabzusetzen. Überdies durften Lehrstellen nicht einfach wieder gekürzt werden. Dies war nur möglich, wenn in fünf aufeinanderfolgenden Jahren die Durchschnittsanzahl der Schüler_innen es erlaubte, nur eine Lehrkraft zu beschäftigen. ${ }^{29}$

Die schon im "Reichsvolksschulgesetz" festgelegte "Gruppierung der Schuljugend in Abtheilungen oder Classen"30 wurde durch die Übergangsbestimmungen vom 12. Juli 1869 genauer geregelt und später in den Lehrplänen verankert. Die grundlegendste Bestimmung lautete, dass Klassen, in denen Kinder unterschiedlichen Alters unterrichtet wurden, in Abteilungen zu gliedern waren. ${ }^{31}$ So konnte sich die Lehrperson der einen Abteilung widmen, während die andere mit "ruhiger" Arbeit beschäftigt war. Die Regelungen lauteten wie folgt: Einklassige Schulen waren in drei Abteilungen zu gliedern, dabei sollte die erste Abteilung dem ersten Schuljahr entsprechen; die zweite Abteilung fasste Schüler_innen des zweiten und dritten Schuljahres und die dritte Abteilung war für Schüler_innen des vierten, fünften und sechsten Schuljahres vorgesehen. In zweiklassigen Schulen bildeten die Schüler_innen der ersten drei Schuljahre die erste Klasse, die Schüler_innen der letzten drei Schuljahre die zweite. Jede Klasse war in zwei bis drei Abteilungen zu unterteilen. ${ }^{32}$ Diesem Muster folgend wurden alle Grundschultypen bis hin zur sechsklassigen Volksschule organisiert. Für ungeteilte einklassige Schulen wurde in dem vom "Ministerium für Cultus und Unterricht" als Vorlage ausgegebenen Lehrplan empfohlen, nicht immer alle Schüler_innen gleichzeitig die Schule besuchen zu lassen. ${ }^{33}$ Die Lehrperson sollte sich in Abwesenheit einer oder mehrerer Abteilungen den anderen Abteilungen intensiver widmen. Somit erklärt

27 Gemäß §16 Uebergangsbestimmungen zur Durchführung des Volksschulgesetzes i. d. F. 12. Juli 1869, RGBI. Nr.130, [http://alex.onb.ac.at/cgi-content/alex?aid=rgb\&datum=1869\&page=486\&size=45], eingesehen 9.3.2018.

28 \$11 Reichsvolksschulgesetz.

29 Politische Schulverfassung, S. 141; §11 Reichsvolksschulgesetz.

30 §7 Reichsvolksschulgesetz.

$31 \S 17$ Übergangsbestimmung.

32 Ebd., §\$18-19.

33 Also Schulen mit ganztägigem Unterricht, im Unterschied zu geteilten einklassigen Schulen, an denen Halbtagsunterricht abgehalten wurde. 
sich auch, warum im Lehrplan von 1874 für diese Schulstufe für die erste Abteilung 19 Wochenstunden vorgesehen waren, während die zweite Abteilung 25 und die dritte Abteilung 28 Stunden enthalten sollten. ${ }^{34}$

Wie bereits 1868 durch das „Schule-Kirche-Gesetz" festgelegt, war der Religionsunterricht der einzige Bereich der Volksschulbildung, der noch der Kirchenbehörde unterstand und somit von Geistlichen abgehalten wurde. ${ }^{35}$ Das "Reichsvolksschulgesetz" legte fest, dass die Kirche über die Lernziele und die Verteilung des Lehrstoffes bestimmen konnte. Die Anzahl der Wochenstunden, die für den Religionsunterricht verwendet würden, wurde aber durch den allgemeinen Lehrplan festgelegt. Die Kirchenbehörde musste bekanntgeben, welche Inhalte sie im Unterricht behandeln wollte. Verfügungen, die nicht mit der allgemeinen Schulordnung vereinbar waren, waren von der Schulleitung oder von der Bezirksschulaufsicht abzuweisen. ${ }^{36}$ Mit diesem Paragraphen untermauerte das "Reichsvolksschulgesetz" klar die Bestimmungen von 1868 und verdeutlichte, welche Stellung die Kirche von nun an im österreichischen Grundschulwesen spielen sollte. Diese Trennung wurde vielerorts zu einem der Hauptgründe für den Widerstand gegen das "Reichsvolksschulgesetz".

Im Gegensatz zur Volksschule war es die Aufgabe der Bürgerschule, „denjenigen, welche eine Mittelschule nicht besuchen, eine über das Lehrziel der allgemeinen Volksschule hinausreichende Bildung zu gewähren." ${ }^{37}$ Der Fächerkanon unterschied sich in vielen Punkten von dem der allgemeinen Volksschule. Während einige Unterrichtsgegenstände nur getrennt voneinander unterrichtet wurden, kamen andere Fächer komplett neu hinzu. Die Schulkinder an Bürgerschulen erhielten Unterricht in

„Religion, Sprache und Aufsatzlehre, Geographie und Geschichte mit besonderer Rücksicht auf das Vaterland und dessen Verfassung, Naturgeschichte, Naturlehre, Arithmetik, Geometrie, Buchhaltung, Freihandzeichnen, Geometrisches Zeichnen, Schönschreiben, Gesang und Leibesübungen."38

Wie schon in den Bestimmungen für Volksschulen war auch in der Bürgerschule für Mädchen zusätzlicher Unterricht in „Weiblichen Handarbeiten und Haushaltskunde“"39 vorgesehen. Durch das „Reichsvolksschulgesetz“ wurden zwei Formen der Bürgerschule festgelegt. Zum einen gab es die selbständigen dreiklassigen Bürgerschulen. Diese schlossen an die Volksschule an und gaben den Schüler_innen die Möglichkeit, nach dem fünften Jahr an die Bürgerschule zu wechseln. Zum anderen war es möglich, „die allgemeine Volksschule so einzurichten, daß sie zugleich die Aufgaben der Bürger-

34 Übergangsbestimmung, Anhang, S. 459; K. k. Unterrichtsminister, Lehrplan für ungetheilte einclassige Volksschulen (mit ganztägigem Unterrichte). Veröffentlicht durch Verordnung des k. k. Unterrichtsministers vom 18. Mai 1874. Z.6549, Wien 1874, S. 2.

35 In Orten, in denen es keinen Geistlichen gab, der den Religionsunterricht regelmäßig abhalten konnte, konnte auch der Lehrer - nach Absprache mit der Kirchenbehörde - die Schüler_innen in seiner Konfession unterrichten.

$36 \$ 5$ Reichsvolksschulgesetz.

37 Ebd., $\$ 17$.

38 Ebd.

39 Ebd. 
schule lösen kann."40 In diesen Fällen schloss die Bürgerschule direkt an die Volksschule an, ein Schulwechsel war nicht vorgesehen. Welcher Bürgerschultypus an einem Ort errichtet werden sollte, wurde von denjenigen entschieden, die die Schule erhielten. ${ }^{41}$

Die meisten Kinder, die eine Bürgerschule besuchten, stammten aus der städtischen Mittelschicht. Die primäre Aufgabe dieser Schulform war es, die Schüler_innen auf die Ausübung von - hauptsächlich kaufmännischen oder handwerklichen - Berufen vorzubereiten. Durch die Novelle von 1883 wurde das Aufgabenfeld der Bürgerschule erweitert und sie gewann an Wichtigkeit.

Dass dem Schulbesuch ein eigener Abschnitt im „Reichsvolksschulgesetz" gewidmet wurde, lässt sich nur dadurch erklären, dass es in diesem Bereich bisher kaum Regelungen gegeben hatte, beziehungsweise, dass sich niemand an die bestehenden Bestimmungen gehalten hatte. Durch das „Reichsvolksschulgesetz" wurde die Schulpflicht von zuvor sechs auf nun acht Jahre erhöht. Ein Austritt aus der Schule war außerdem nur dann möglich, wenn "die Schüler die für die Volksschule vorgeschriebenen nothwendigen Kenntnisse [...] besitzen." 42 Von der Pflicht, die Volksschule zu besuchen, waren nur Knaben, die eine höhere Schule besuchten sowie "Kinder, denen ein dem Unterrichtszwecke oder Schulbesuche hinderliches geistiges oder schweres körperliches Gebrechen anhaftet"43, befreit. Eine weitere Ausnahme bildeten Kinder, die zu Hause oder in privaten Anstalten unterrichtet wurden. Eltern hatten von nun an außerdem die Aufgabe, sicherzustellen, dass ihre Kinder die Schule auch regelmäßig besuchten. Sollten sie dieser Pflicht nicht nachkommen, sah das „Reichsvolksschulgesetz" die Anwendung von Zwangsmitteln vor. Genauere Bestimmungen dazu waren in der Landesgesetzgebung zu finden. ${ }^{44}$ In Kärnten sah die Landesgesetzgebung beispielsweise vor, dass das Schulversäumnis mindestens einmal pro Monat von der Schule kontrolliert werden musste. Bei Verstößen waren die Eltern oder Erziehungsberechtigen zu kontaktieren, um gegen die Nachlässigkeit von ihrer Seite einzuschreiten. ${ }^{45}$

\subsection{Durchführung, Kritik und Widerstand}

Bereits vor der Debatte rund um das „Reichsvolksschulgesetz“ war das Verhältnis zwischen Liberalen und Konservativen sehr angespannt. Grund dafür war unter anderem das „Konkordat von 1855“ beziehungsweise die Forderung der Liberalen, dieses zu lösen. Dass durch das Reichsvolksschulgesetz das österreichische Schulwesen unter staatliche Aufsicht gestellt werden sollte, regte diesen Konflikt erneut an. ${ }^{46}$

40 Reichsvolksschulgesetz, §18

41 Ebd., $\$ 18$.

42 Ebd., $\$ 21$.

43 Ebd., $\$ 23$.

44 Ebd., $\$ 24$

45 Boyer, Elementarschulen, S. 285.

46 Karl Vocelka, Verfassung oder Konkordat? Der publizistische und politische Kampf der österreichischen Liberalen um die Religionsgesetze des Jahres 1868 (Studien zur Geschichte der österreichisch-ungarischen Monarchie XVII), Wien 1978, S. 51 und S. 166-167. 
Bei der Abstimmung zum „Reichsvolksschulgesetz“ kam es im Abgeordnetenhaus zu einer heftigen Debatte, woraufhin 65 Abgeordnete den Sitzungssaal verließen. Bei der folgenden Abstimmung - in Abwesenheit der 65 Herren - erhielt das "Reichsvolksschulgesetz" eine überwältigende Mehrheit von 111 zu vier Stimmen. Nach der anschließenden Zustimmung des Herrenhauses und der Sanktionierung des Kaisers trat das „Reichsvolksschulgesetz" am 14. Mai 1869 in Kraft; seine Bestimmungen sollten schon ab Beginn des Schuljahres 1869/70 umgesetzt werden. ${ }^{47}$

Damit die Bestimmungen des „Reichsvolksschulgesetzes“ auch in der Praxis umgesetzt werden konnten, waren Ausführungsgesetze von den Landtagen der einzelnen Länder notwendig. In den meisten Gebieten der Monarchie funktionierte die Erlassung dieser Gesetze einwandfrei. Ende 1870 waren nur noch Tirol und die Stadt Triest ausständig. ${ }^{48}$ Um den Übergang zu den neuen Bestimmungen zu erleichtern und "mit Rücksicht darauf, dass einzelne Landesgesetze bezüglich der Volksschulen noch nicht zustande gekommen“49 waren, wurde am 20. August 1870 die „Schul- und Unterrichtsordnung für die allgemeinen Volksschulen" erlassen. In ihr wurden grundlegende Bestimmungen wie etwa das Schulversäumnis, der Beginn des Schuljahres sowie das Verbot der körperlichen Züchtigung geklärt. Außerdem wurden die Lehrziele für die Unterrichtsgegenstände detailliert beschrieben, da die Lehrpläne, die von den Lehrerbildungsanstalten ausgearbeitet wurden, noch nicht bereitstanden. ${ }^{50}$

So innovativ und längst überfällig das „Reichsvolksschulgesetz” aus heutiger Sicht auch erscheinen mag, wurde es im Zuge seiner Verabschiedung heftig kritisiert. Die Kritik kam dabei von verschiedenen Seiten und betraf unterschiedliche Aspekte des Gesetzes. Ein Punkt, der von der Opposition - bestehend aus Föderalist_innen, Konfessionellen und Ständisch-Konservativen - bemängelt wurde, war, dass der Staat versuche, die Schule nach seiner politischen Auffassung zu gestalten. Es wurde argumentiert, dass dabei der Wille der Eltern missachtet werden würde. Die Schule sollte "die gesellschaftliche Organisation der häuslichen Erziehung"51 bleiben. Das neue Schulgesetz hingegen wurde als Eindringen des Staates in das elterliche Erziehungsrecht gewertet. Die politische Bildung und Erziehung der Kinder war zu dieser Zeit Aufgabe und Privileg der Eltern. Die religiöse Erziehung auf der anderen Seite war bis dahin eindeutig Aufgabe der Schule beziehungsweise der Kirche, die ja den Großteil der Schulen führte, gewesen. Dass die Aufsicht über die Schulen und die Leitung des Unterrichts - abgesehen vom Religionsunterricht - der katholischen Kirche nun entzogen werden sollten, führte ebenfalls zu viel Unmut. Das pädagogische und didaktische Prinzip des „Reichsvolksschulgesetzes“ selbst wurde kaum kritisiert. Ein Punkt,

47 Engelbrecht, Geschichte 4, Anmerkung 53, S. 388; Boyer, Elementarschulen, S. 289.

48 Boyer, Elementarschulen, S. 289.

49 Verordnung, womit eine Schul- und Unterrichtsordnung für die allgemeinen Volksschulen erlassen wird i. d. F. 20. August 1870, RGBI. Nr. 105, Präambel [http://alex.onb.ac.at/cgi-content/alex?aid=rgb\&datum $=18700004 \&$ sei te=00000219], eingesehen 14.3.2018.

50 Verordnung, womit eine Schul- und Unterrichtsordnung für die allgemeinen Volksschulen erlassen wird i. d. F. 20. August 1870, RGBI. Nr. 105, [http://alex.onb.ac.at/cgi-content/alex?aid=rgb\&datum=18700004\&seite=00000219], eingesehen 14.3.2018.

51 Engelbrecht, Geschichte 4, S. 116; Friedrich Jellouschek, Das Reichsvolksschulgesetz, in: Erziehung und Unterricht 5 (1969), S. 289-305, hier S. 301; Vocelka, Verfassung oder Konkordat?, S. 166. 
der jedoch häufig thematisiert wurde, war, dass die Inhalte, die den Schulkindern nun vermittelt werden sollten, für die Volksschule viel zu umfangreich waren. Außerdem wären die Lehrinhalte zu verweltlicht und würden somit nur eine Seite der Lebenswelt der Kinder widerspiegeln. Durch dieses Missverhältnis wäre die "Menschwerdung" der Schüler_innen gefährdet. ${ }^{52}$ Ein wesentlicher Aspekt, der viele Menschen erzürnte, war, dass der Staat die umfassende Reformierung des Grundschulwesens angeordnet hatte, sich selbst aber kaum an den entstehenden Kosten beteiligen wollte. Lehrer_innen sollten nicht nur besser ausgebildet, sondern auch besser bezahlt werden und die Ausstattung der Klassenzimmer und Schulgebäude sollte erweitert werden. Die Finanzierung hatten die Gemeinden beziehungsweise die Schulbehörden zu tragen. ${ }^{53}$ Sowohl bei der Landbevölkerung als auch bei Fabrikbesitzer_innen war die Verlängerung der Schulpflicht um zwei Jahre nicht gerne gesehen. Schließlich bedeutete das, dass man zwei weitere Jahre auf die Arbeitskraft der Kinder verzichten musste. „Die Ausdehnung der Schulpflicht [...] wurde von der bäuerlichen Bevölkerung [...] als schwere Belastung empfunden. Dazu kam, daß die Lehrpläne im allgemeinen viel zu wenig Rücksicht auf die Bedürftnisse [sic!] der Landschule genommen hatten." ${ }^{45}$

Der Widerstand gegen das neue Gesetz gestaltete sich recht unterschiedlich. Im Folgenden wird Tirol näher betrachtet, da die Ablehnung dort ein besonders großes Ausmaß annahm.

Dort weigerte man sich, die Gesetze zu erlassen, die für das Inkrafttreten des „Reichsvolksschulgesetzes" nötig waren, da seine Bestimmungen sowohl von Seiten der Kirche als auch von Seiten der Bevölkerung auf enormen Widerstand trafen. Die Ablehnung der Neugestaltung des Elementarschulwesens hatte keine bestimmte Ursache. Vielmehr war es die Verknüpfung einer Vielzahl von Faktoren, die nicht mit den bisherigen Gegebenheiten in Tirol zusammenpassten, die dazu führte, dass der Tiroler Landtag sich weigerte, die entsprechenden Gesetze zu erlassen. Zunächst einmal war das Grundschulwesen in Tirol viel enger an die Kirche gebunden als in anderen Teilen der Monarchie. Daraus resultierte einerseits, dass die religiöse Erziehung für die Bevölkerung klar Teil der schulischen Erziehung und somit die Kirche als logischer Vorstand der Schule gesehen wurde. Andererseits trug die Kirche in großen Teilen Tirols auch erheblich zur Finanzierung von öffentlichen Schulen bei, sodass in einigen Gemeinden der Erhalt von Schulen ohne diese Mittel nicht möglich schien. Erschwerend kam hinzu, dass der Tiroler Landtag vor allem mit Konservativen besetzt war und unter der Führung des Fürstbischofs von Brixen stand. In zahlreichen Sitzungen konnte man sich nicht auf ein Ausführungsgesetz zum „Reichsvolksschulgesetz" einigen. Die Argumente waren - ähnlich jenen, die auch im Rest der Monarchie vorgebracht worden waren - zu lange Schulpflicht, die Interkonfessionalität sowie eine zu hohe Anforderung an die Ausbildung der Lehrer_innen. Im Gegensatz zu anderen Regionen sah man in Tirol aber die politische sowie die religiöse Einheit des Landes gefährdet und bestand dar-

Engelbrecht, Geschichte 4, S. 116

\$5 Reichsvolksschulgesetz.

Franz Haselwanter, Die Entwicklung des Volks- und Bürgerschulwesens. In den Jahren 1910 bis 1927, Innsbruck 1985, S. 39. 
auf, dass das "Reichsvolksschulgesetz“ nicht mit dem Tiroler Landesrecht vereinbar sein sollte. Als die Regierung mit der Schul- und Unterrichtsordnung für die allgemeinen Volksschulen 1870 versuchte, die Grundsätze des "Reichsvolksschulgesetzes" auch in Tirol wirksam zu machen, stieß sie auf enorme Gegenwehr von Seiten der Tiroler Bevölkerung. In einigen Gemeinden wurden die Schulinspektoren, die die Umsetzung der neuen Richtlinien überprüfen sollten, gewaltsam daran gehindert, das Schulgebäude zu betreten. In anderen Gemeinden riefen die Pfarrer die Eltern dazu auf, ihre Kinder am Tag der Schulinspektion nicht in die Schule zu schicken. Mancherorts fand der Schulinspektor daher leere Klassenräume vor. ${ }^{55}$

Erst 1892 verfasste und verabschiedete die Tiroler Landesregierung sowohl ein Schulaufsichtsgesetz als auch ein Gesetz, das die allgemeinen Volksschulen betraf. Beide stimmten zwar noch immer nicht in allen Punkten mit dem „Reichsvolksschulgesetz" überein, wurden aber vom Ministerium abgesegnet.

In Vorarlberg gab es zwar keinen offenen Widerstand gegen das „Reichsvolksschulgesetz", trotzdem war es auch hier ein langer Weg, bis letztendlich alle Bestimmungen umgesetzt wurden. Die Vorarlberger Bevölkerung hatte die Verlängerung der Schulpflicht zwar prinzipiell befürwortet, wünschte sich aber mit Berücksichtigung der örtlichen Verhältnisse, den Schulbesuch in den Sommermonaten etwas flexibler zu gestalten. So wurde 1870 für Tirol und Vorarlberg eine Sonderregelung getroffen, die es allen zwölf- bis vierzehnjährigen Schulkindern eines Ortes erlaubte, der Schule während des Sommers fernzubleiben. In manchen Gemeinden wurde dieses Entgegenkommen aber zu frei interpretiert und der Landesschulrat musste den Schulbesuch dieser Altersgruppe durch Androhung und Verhängung von Strafgeldern oder Freiheitsstrafen erzwingen. Es dauerte bis 1880, bis man den Besuch der Winterschule für alle schulpflichtigen Kinder endlich durchsetzen konnte. ${ }^{56}$

\subsection{Die Entwicklung des Grundschulwesens nach dem "Reichsvolksschulgesetz"}

Durch das „Reichvolksschulgesetz" war bestimmt worden, dass für Bürgerschulen und alle Kategorien der Volksschule einheitliche Lehrpläne erstellt werden sollten. Am 18. Mai 1874 traten die insgesamt zehn Lehrpläne endgültig in Kraft. ${ }^{57}$ Es war das erste Mal in der Geschichte der österreichischen Volksschule, dass es einheitliche Lehrpläne gab. Ihre Aufgabe war es, den Unterrichtsstoff zu begrenzen, also zu reglementieren, was mindestens und maximal unterrichtet werden sollte. Dabei wurden die pädagogischen Grundsätze der Zeit berücksichtigt. Der Inhalt des Unterrichts sollte an das Alter der Kinder angepasst werden. ${ }^{58}$ Auf der ersten Seite jedes Lehrplans fand man eine Stundentafel, die nicht nur über die Anzahl und Verteilung der Stunden auf die jeweiligen Fächer, sondern auch über die Aufteilung der Schüler_innen in Klassen und Abtei-

\footnotetext{
55 Boyer, Elementarschulen S. 289; Engelbrecht, Bildungswesen 4, S. 117.

56 Boyer, Elementarschulen, S. 345-346.

57 Für geteilte und ungeteilte einklassige Volksschulen, zwei- bis siebenklassige Volksschulen sowie je ein Lehrplan für Knaben- und Mädchenbürgerschulen.

58 Boyer, Elementarschulen, S. 318.
} 
lungen Auskunft gab. Auf den folgenden Seiten standen genauere Auskünfte bezüglich der Lehrinhalte für die einzelnen Fächer geschrieben und in welchem Schuljahr die Themen behandelt werden sollten. Die Lehrstoffangaben bezogen sich jeweils auf die einzelnen Abteilungen, nicht auf die gesamte Klasse. Die Aufteilung der einzelnen Klassen in Abteilungen verlief nach einem eindeutigen Muster. Bei zunehmender Klassenzahl wurden die unteren Schuljahre zuerst als eigenständige Klassen geführt. Das erste Schuljahr als eigenständige Klasse zu führen war ab der vierklassigen Volksschule möglich. Die restlichen Schuljahre wurden in Klassen zusammengefasst und dort nach Abteilungen unterschieden. Im Regelfall gab es zwei Abteilungen pro Klasse. ${ }^{59}$ Eine Besonderheit, die man heute nicht mehr in Lehrplänen findet, sind die Hinweise auf Methoden in einigen Lehrgegenständen. In Erdkunde wurde für die vierte Klasse beispielsweise die Behandlung des Heimatlandes und die "graphische Darstellung desselben an der Schultafel"60 vorgeschrieben. Weitere methodische Vorschriften gab es in den Realien, Gesang und Zeichnen. Für das Lesen-Lernen gab der Lehrplan gleich ein ganzes Methodensystem vor. ${ }^{61}$

1883 kam es zur Novellierung des "Reichsvolksschulgesetzes". Die Liberalen, die die treibende Kraft hinter dem „Reichsvolksschulgesetz" gewesen waren, verloren im Laufe der 1870er und 1880er immer mehr an Macht. Zudem wollte auch die Kritik an der neuen Schulform nicht verstummen. So kam es, dass 1882 eine Regierungsvorlage zur Novellierung des „Reichsvolksschulgesetzes" eingebracht wurde. Sowohl im Herrenals auch im Abgeordnetenhaus wurde heftig debattiert. Jede Kritik, die in den letzten Jahren am „Reichsvolksschulgesetz" geübt worden war, wurde erneut hervorgebracht. Insgesamt gab es vierzig Änderungsvorschläge, wobei der erste und am hitzigsten diskutierte die Änderung der Aufgabe der Volksschule von "sittlich-religiöser" Erziehung zu "religiös-sittlicher" war. Letztendlich konnte man sich hier aber nicht einigen und so blieb es bei der ursprünglichen Formulierung. Nachdem der Inhalt der Novelle in beiden Häusern gründlich besprochen und geprüft worden war, konnte sie am 2. Mai 1883 beschlossen werden. Sie änderte 25 Paragraphen des „Reichsvolksschulgesetzes" ganz oder teilweise ab. Leichte Veränderungen gab es in der Auflistung der Lehrgegenstände für die allgemeine Volksschule. Aus "Schreiben“ wurde "Lesen und Schreiben" und aus "das Wissenswerteste aus Naturkunde, Erdkunde und Geschichte mit besonderer Rücksichtnahme auf das Vaterland und dessen Verfassung" wurde "das für die Schüler Faßlichste und Wissenswertheste aus der Naturgeschichte, Naturlehre, Geographie und Geschichte mit besonderer Rücksichtnahme auf das Vaterland und dessen Verfassung"62. Derselbe Paragraph brachte aber auch wesentliche Neuerungen. Der Turnunterricht war von nun an nur für Knaben obligat; Mädchen durften nur

59 Boyer, Elementarschulen, S. 321; Eine Ausnahme bilden einklassige Volksschulen. Diese wurden in drei Abteilungen geteilt.

60 Lehrplan für siebenclassige Volksschulen Veröffentlicht durch Verordnung des k. k. Unterrichtsministers vom 18. Mai 1874. Z. 6549, Wien 1874, S. 7.

61 Boyer, Elementarschulen, S. 321-322.

62 Boyer, Elementarschulen, S. 354; Gemäß $\$ 3$ Novelle des Gesetzes vom 14. Mai 1869, abgeändert werden i. d. F. 2. Mai 1883, [http://alex.onb.ac.at/cgi-content/alex?aid=rgb\&amp;datum=1883\&amp;size=45\&amp;page=225], eingesehen 12.3.2018. 
turnen, wenn ihre Eltern sie dafür anmeldeten. Den Schulen stand es immer noch frei, den Unterricht um andere Gegenstände zu erweitern, wenn genügend Lehrpersonal vorhanden war. Neu war allerdings, dass darauf hingewiesen wurde, dass eine solche Erweiterung vor allem in einer anderen Landessprache sinnvoll sei. ${ }^{63}$ Der Teil von $§ 7$ des "Reichsvolksschulgesetzes”, der die Trennung der Geschlechter in der Volksschule empfahl, wurde durch die Novelle gestrichen.

Die Bürgerschule erfuhr durch die Novellierung von 1883 eine Änderung ihres Lehrauftrages. Dieser lautete nun:

„Die Bürgerschule hat eine über das Lehrziel der allgemeinen Volksschule hinausreichende Bildung, namentlich mit Rücksicht auf die Bedürfnisse der Gewerbetreibenden und der Landwirthe zu gewähren. Dieselbe vermittelt auch die Vorbildung für Lehrerbildungsanstalten und für jene Fachschulen, welche eine Mittelschulvorbildung nicht voraussetzen." ${ }^{164}$

Das Lehrziel der Bürgerschule war also erweitert worden, wodurch ihre Bestimmung und auch ihre "Zielgruppe" klarer wurden. Außerdem wurde die Organisation der Bürgerschule umstrukturiert. Es war nicht mehr vorgesehen, dass Volksschulen zu Bürgerschulen erweitert wurden und als solche fungierten. Die Bürgerschule bestand nun aus drei eigenständigen Klassen und bildete eine eigenständige Schulform. Es war jedoch noch möglich, eine Volks- und eine Bürgerschule unter einer Leitung zu verbinden. Diese trug dann die Bezeichnung „Allgemeine Volks- und Bürgerschule“. Die Lehrgegenstände der Bürgerschule änderten sich kaum. Auch hier kam es zu Umbenennungen von Unterrichtsgegenständen. Wie auch in der Volksschule war der Turnunterricht in der Bürgerschule von nun an für Mädchen nicht mehr obligat.65

Zu einem allgemeinen Entgegenkommen kam es bezüglich des Schulbesuches von Schüler_innen während der letzten beiden Schuljahre. Wie bereits 1870 in der Sonderregelung für Tirol und Vorarlberg festgehalten, konnten nun Schüler_innen nach dem sechsten Schuljahr für einen gewissen Zeitraum vom Unterricht freigestellt werden, wenn ihre Eltern darum ansuchten. Diese Erleichterung bestand aus einer „Einschränkung des Unterrichts auf einen Theil des Jahres, oder auf halbtägigen Unterricht oder auf einzelne Wochentage" und konnte auch für "Kinder ganzer Schulgemeinden auf dem Lande" gewährt werden. In jedem Fall mussten die Kinder aber die vorgegebenen Lehrziele nach Beendigung ihrer Schulpflicht erreicht haben. Die Schulbesuchserleichterung wurde von vielen Gemeinden in Anspruch genommen. In Niederösterreich wurde diese Regelung beispielsweise von 652 der insgesamt 906 Gemeinden beansprucht. ${ }^{66}$ Mit dieser Regelung versuchte die Regierung, der Bevölkerung - vor allem in ländlichen Gebieten - entgegenzukommen. Nun stand den Eltern die Arbeitskraft der

66 \$21 Novelle; Auguste Fickert, Der Stand der Frauenbildung in Österreich, in: Gertrud Bäumer/Helene Lange (Hrsg.), Handbuch der Frauenbewegung. Der Stand der Frauenbildung in den Kulturländern, Berlin 1902, S. 160190, hier S.170. 
Kinder während der Erntezeit zur Verfügung. In den anderen Monaten konnten sie die schule besuchen.

\section{Geschlechtsspezifische Differenzierung im österreichischen Grundschulwesen}

Das Volksschulwesen bietet sich für eine Analyse der geschlechtsspezifischen Differenzierung aus mehreren Gründen besonders an. Zum einen war es der einzige Teil des österreichischen Schulsystems, in dem auch Mädchen von den Reformen, die das Schulwesen ab der zweiten Hälfte des 19. Jahrhunderts erfuhr, betroffen waren. ${ }^{67}$ Zum anderen stellt es die einzige Schulform dar, die gleichermaßen von Knaben als auch von Mädchen besucht wurde und in der verhältnismäßig die wenigsten Unterschiede zwischen den Geschlechtern gemacht wurden. Trotzdem machen sich bereits hier eindeutig die gesellschaftlichen Stereotype der Zeit bemerkbar. Mädchen, denen für lange Zeit nach der Volks- beziehungsweise Bürgerschule kaum Bildungswege offenstanden, wurden gezielt auf ihre Rollen als Hausfrauen oder Dienstmädchen vorbereitet. In den Bürgerschulen wurde die geschlechtsspezifische Differenzierung noch offensichtlicher.

Mit dem Inkrafttreten des „Reichsvolksschulgesetzes“ kam es zu einer Diskussion darüber, ob die Trennung von Mädchen und Knaben in der Grundschule sinnvoll sei. Das Thema Koedukation stand nun zur Debatte. Gemischte Volksschulen wurden nicht zwingend als Notbehelfe für Gemeinden, die die Errichtung und den Unterhalt eigener Schulen für die Geschlechter nicht finanzieren konnten, gesehen. Das „Reichsvolksschulgesetz" selbst griff den Gedanken der Koedukation nicht auf, jedoch fand er in der Gesellschaft Anklang. 1874 wurde beispielsweise im Salzburger Volksblatt argumentiert, dass eine gemeinsame Ausbildung von Mädchen und Knaben auch Vorteile mit sich brächte. Die gemeinsame Erziehung der Geschlechter bis zum Alter von zwölf Jahren sollte dazu führen, dass "sich die Ungebärdigkeit der Knaben durch die Anwesenheit der Mädchen abschleife, und übermäßige Empfindlichkeiten der Mädchen sich gar nicht erst ausbilden könnten." ${ }^{168}$

\subsection{Bildung für Mädchen? Das Gesellschaftsbild im 19. und 20. Jahrhundert}

Seit jeher wurde in Sachen Bildung ein Unterschied zwischen den Geschlechtern gemacht, wobei diese geschlechtsspezifische Differenzierung zu keiner Zeit zu Gunsten von Mädchen und Frauen ausfiel. Seitdem der Rationalität in der Gesellschaft aber eine immer wichtigere Rolle zugeschrieben wurde, musste diese Unterscheidung auch wissenschaftlich belegt werden. Und so ist es nicht verwunderlich, dass "die Natur der Frau" in der Entwicklung von Bildungskonzepten im 19. Jahrhundert ein wichtiger Aspekt wurde. ${ }^{69}$

67 Friedrich, Hatte Vater Staat nur Stieftöchter?, S. 304.

68 Friedrich, „Ein Paradies ist uns verschlossen...., S. 61.

69 Ebd., S. 210. 
Schon seit der Aufklärung hatte sich das Bild manifestiert, dass der "natürliche" Aufgabenbereich der Frau nur den Haushalt und die Versorgung der Kinder umfasste. Allgemein wurde davon ausgegangen, "daß das weibliche Geschlecht keinen anderen Lebenszweck habe, als den, in der Ehe einem Gatten die Lasten der häuslichen Arbeit abzunehmen." ${ }^{\prime 70}$ Bildung, die über das Wissen, das in der Volksschule vermittelt wurde, hinausreichte, war dafür nicht notwendig. Der Schriftsteller Joachim Heinrich Campe fürchtete beispielsweise, dass Mädchen „den Weg in Küche und Kinderstube nicht mehr fänden"71, wenn sie intellektuelle Bildung erhielten. Das Ziel einer "möglichst guten Ausbildung aller individuellen Anlagen“ entwickelte sich für Knaben schon im frühen 19. Jahrhundert. Für Mädchen wurde im Gegensatz dazu noch immer das Haus als "günstigster und maßgeblicher Erziehungsort" gesehen, an dem sie durch Nachahmung alles erlernen konnten, was sie für ihr späteres Leben benötigten. Über eine Verbesserung der Ausbildung von Mädchen wurde erst diskutiert, als erkannt wurde, dass Frauen durch ihren Einfluss auf ihre Männer und Kinder sehr wohl auch Einfluss auf die Gesellschaft hatten. Im Zuge dessen wurde über die Zulassung von Mädchen zu Gymnasien diskutiert. Noch 1891 argumentierte ein Abgeordneter dazu, dass es „eine Entgleisung und ein Fehler gegen die Natur, ja sogar ein Eingriff in die physische Entwicklung" der Frau sei, wenn man sie von ihrer natürlichen Pflicht abbrächte. Weiter meinte er, dass es nur wenige Frauen gäbe, die die Arbeit, die nach einem Studium folge, einem liebenden Ehemann vorziehen würden. ${ }^{72}$ Allgemein war noch zu Beginn des zwanzigsten Jahrhunderts die Überzeugung weit verbreitet, dass der weibliche Geist prinzipiell nicht so belastbar wäre wie der männliche. Auch von denjenigen, die für eine verbesserte Mädchenbildung eintraten, wurde oft argumentiert, dass Mädchen das, "was ihnen die Knaben an schnellerer Auffassungsgabe voraus hätten"73, durch Fleiß wieder wettmachen würden. Selbst sie konnten sich also nicht von dem Vorurteil lösen, dass ein Unterschied im Auffassungsvermögen von Mädchen und Knaben bestand. Die „Eigenart des weiblichen Geschlechts“ blieb bis zum Ende der Monarchie ein wichtiges Argument in der Diskussion um Mädchenbildung. Besonders von ministerieller Seite wurde sie immer wieder betont, um eine Anpassung des Unterrichts für Mädchen an deren spezielle Bedürfnisse zu rechtfertigen. ${ }^{74}$

70 Julie Burow, Ueber die Erziehung des weiblichen Geschlechts, 1856, in: Günter Häntzschel (Hrsg.), Bildung und Kultur bürgerlicher Frauen 1850-1918. Eine Quellendokumentation aus Anstandsbüchern und Lebenshilfen für Mädchen und Frauen als Beitrag zur weiblichen literarischen Sozialisation, Tübingen 1986, S.88-107, hier S. 89.

71 Friedrich, „Ein Paradies ist uns verschlossen...., S. 210.

72 Margret Friedrich, Hatte Vater Staat nur Stieftöchter?, S. 332-333; Stenographische Protokolle des Abgeordnetenhauses, 62. Sitzung der XI Session vom 3. November 1891, S. 2881, zit. in: Margret Friedrich, „Ein Paradies ist uns verschlossen...." Zur Geschichte der schulischen Mädchenerziehung in Österreich im "langen“ 19. Jahrhundert, Wien-Köln-Weimar 1999, S. 212; Friedrich, Ein Paradies ist uns verschlossen...," S. 213.

73 Ebd., S. 216

74 Ebd., S. 333; Worin diese Eigenart genau bestand wurde nirgends festgehalten. Offensichtlich ging man von einer allgemeinen Einigkeit zu dieser Thematik aus, sodass der Begriff keiner genaueren Definition bedurfte. Bei den Diskussionen um die Ziele der Mädchenbildung zeigte sich aber, dass eine solche Einigkeit nicht bestand. 


\subsection{Geschlechtsspezifische Unterschiede in Volksschulen}

Schon die unter Maria Theresia 1774 verfasste „Allgemeine Schulordnung für die deutschen Normal-, Haupt- und Trivialschulen" legte fest, dass Mädchen und Buben ein Recht auf die gleiche Bildung hatten. Die Trennung der Geschlechter in der Schule war trotzdem vorgesehen, um die Mädchen „auch in Nähen, Stricken, und in andern ihrem Geschlechte angemessenen Dingen zu unterweisen."75 Nur in Orten, in denen es nicht möglich war, eine eigene Mädchenschule zu errichten, sollten die Mädchen gemeinsam mit den Knaben unterrichtet werden. Sie sollten „jedoch nicht unter den Knaben, sondern auf eigenen Bänken von denselben abgesondert sitzen"76 und trotzdem in den „für ihr Geschlecht angemessenen Dingen"77 unterrichtet werden.

Auch bei der Reformierung der Volksschule im Jahr 1848 sollten bei der Auswahl der Lehrinhalte nach Franz Exner "die Unterschiede zwischen Stadt- und Landkindern [und] zwischen den Geschlechtern [...] berücksichtig[t]"78 werden. Die Einrichtung von Mädchenschulen wurde erneut empfohlen. De facto besuchten die meisten Kinder aber gemischte Schulen. Trotzdem ist zu beobachten, dass die Anzahl der reinen Mädchenschulen in der Zeit vor dem „Reichsvolksschulgesetz" anstieg. Während 1828 nur rund fünf Prozent aller Grundschulen für Mädchen waren, waren es 1867 bereits fast 13 Prozent. $^{79}$ In den gemischten Schulen wurde noch immer strengstens darauf geachtet, dass die Geschlechter voneinander getrennt wurden. Außerdem ist anzumerken, dass ein gemeinsamer Unterricht nicht mit qualitativ gleichwertigem Unterricht gleichzusetzen ist. Zwar sollten prinzipiell dieselben Lehrmethoden und Schulbücher für Mädchen und Knaben verwendet werden, doch nicht selten wurden die Lerninhalte für Mädchen stark gekürzt. Ziel der Volksschule war es, die Mädchen zu "sorgfältigen Hausfrauen und willigen Dienstbotinnen"80 heranzuziehen. So sollten sie in Mathematik beispielsweise nur die Fähigkeiten erlernen, die nötig waren, um ein Haushaltsbuch zu führen und eine Monats- und Jahresabrechnung zu erstellen. ${ }^{81}$

Prinzipiell galt nach §14 des „Reichsvolksschulgesetzes", dass alle Bestimmungen, die für Knabenschulen sowie gemischte Schulen galten, auch für selbständige Mädchenschulen gelten sollten. ${ }^{82}$ Darunter fielen unter anderem „die Auswahl und Anordnung des Lehrstoffes, die Anzahl der Lehrkräfte und die Anstellung von Lehrerinnen und Unterlehrerinnen." ${ }^{13}$ Dass das bürgerliche Frauenbild ${ }^{84}$ auch einen Einfluss auf die Ausarbeitung des "Reichsvolksschulgesetzes" hatte, zeigt sich schon daran, dass Mädchen

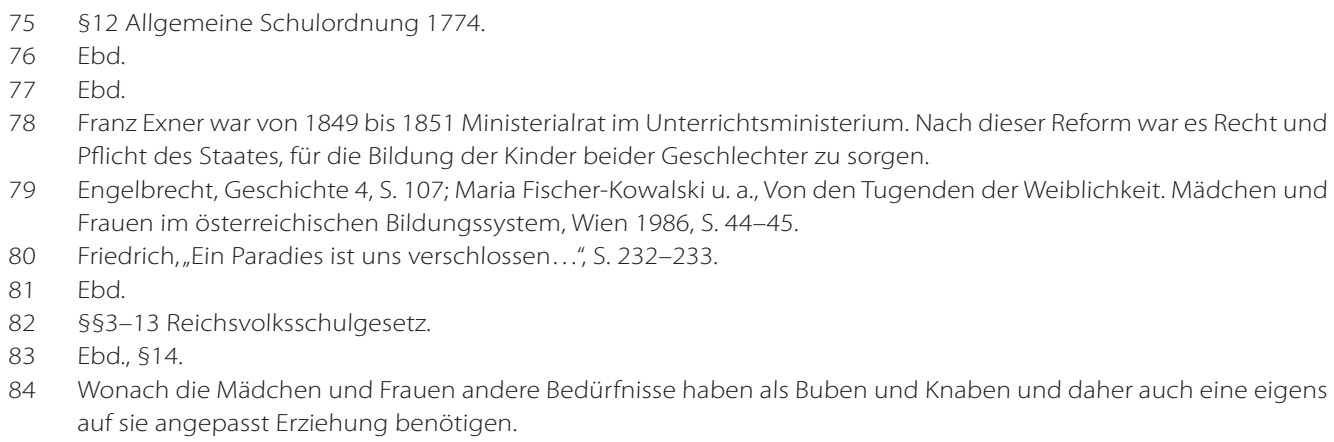

84 Wonach die Mädchen und Frauen andere Bedürfnisse haben als Buben und Knaben und daher auch eine eigens auf sie angepasst Erziehung benötigen. 
weiterhin in Handarbeiten unterrichtet wurden. Die 1874 ausgearbeiteten Lehrpläne zeigen deutlich, dass hier beträchtliche Unterschiede zwischen Mädchen- und Knabenschulen gemacht wurden. Allgemein lässt sich festhalten, je einfacher der Schultyp war, umso weniger Unterschiede wurden in der Stundentafel gemacht, während Mädchen in den in mehrere Klassen unterteilten Volksschulen mehr Stundenkürzungen zur Kenntnis nehmen mussten. So waren in einklassigen Volksschulen nur in „Zeichnung” und "Geometrischer Formenlehre" weniger Stunden vorgesehen, während in einer siebenklassigen Volksschule Mädchen in fünf Fächern, nämlich Zeichnen und Geometrische Formenlehre, Rechnen, Turnen, Erdkunde und Geschichte und Schreiben weniger Unterricht erhielten. Meist steigerten sich die Unterschiede in der Stundentafel von Klasse zu Klasse. ${ }^{85}$

An dieser Stelle soll aber auch erwähnt werden, dass Mädchen, wie durch das „Reichsvolksschulgesetz" vorgesehen, noch zusätzlich Unterricht in „Weiblichen Handarbeiten und Haushaltskunde“ erhielten, wobei „Haushaltskunde“ nicht als eigenständiges Fach unterrichtet wurde, sondern in die „einschlägigen Gegenstände“ integriert werden sollte. ${ }^{86}$ Für ein- bis vierklassige Volksschulen wurde durch die Lehrpläne nicht geregelt, in welchem Umfang "Weibliche Handarbeiten“ unterrichtet werden sollte. Die Entscheidung darüber oblag der betreffenden Schulbehörde. Erst ab der fünfklassigen Volksschule war durch die Stundentafel des Lehrplans festgelegt, wie viele Stunden pro Schuljahr für jede Klasse beziehungsweise Abteilung für den Unterricht in „Weiblichen Handarbeiten" verwendet werden sollten. Durch die Addierung dieser Stunden kamen Mädchen in diesen Schultypen ab der dritten Klasse, in der der Unterricht in „Weiblichen Handarbeiten" eingeführt wurde, insgesamt sogar auf mehr Stunden als ihre männlichen Mitschüler. Erst in der letzten Klasse waren für Mädchen und Knaben wieder gleich viele Unterrichtsstunden vorgesehen. ${ }^{87}$ Auch wenn die Ausbildung in "Handarbeiten" und „Haushaltskunde" bedeutete, dass Mädchen weniger Unterricht in anderen Gegenständen erhielten, ist dies nicht zwingend als Benachteiligung zu verstehen. Es ermächtigte die jungen Frauen dazu, später einem Haushalt vorzustehen und sparsam zu wirtschaften. Somit trug dieser Unterricht zur Eigenständigkeit und späteren Unabhängigkeit der Mädchen bei.

Aber auch inhaltlich waren in einigen Fächern Unterscheidungen zwischen den Geschlechtern vorgesehen. In den Lehrplänen findet man beispielsweise den Hinweis, dass im Rechenunterricht in der letzten Klasse "für die Mädchen Haushaltungs-Rechnungen besondere Berücksichtigung finden"88 sollen. Für "Zeichnen" und, bis zur dreiklassigen Volksschule, "Naturlehre” findet sich in den Lehrplänen die Bemerkung, "Haushaltung und Weibliche Handarbeiten" zu integrieren. ${ }^{89}$ Die Formulierung dieser Bestimmungen lässt auf den ersten Blick darauf schließen, dass es sich um zusätzliche

$86 \$ 3$ Reichsvolksschulgesetz; Beispielsweise in Lehrplan für zweiclassige Volksschulen, in: Lehrpläne für Volks- und Bürgerschulen. Veröffentlicht durch Verordnung des k. k. Unterrichtsministers vom 18. Mai 1874. Z. 6549, Wien 1874, S. 9.

87 In siebenklassigen Volkschulen schon ab der sechsten Klasse.

88 Beispielsweise Lehrplan für zweiclassige Volksschulen, S. 4.

89 Freihandzeichnen in siebenklassigen Volksschulen. 
verstärkte Behandlung dieser Themen und nicht um eine Berücksichtigung auf Kosten anderer Themen handelte. Der einzige Lehrgegenstand, in dem klar formuliert wurde, dass einige Inhalte nicht für Mädchen geeignet sind, war der Turnunterricht. Die im Lehrplan mit einem Sternchen markierten Übungen waren nicht für das Mädchenturnen geeignet. Außerdem gab es Übungen, die Mädchen nur eingeschränkt ausführen mussten. Diese Einschränkungen, wie beispielsweise Liegestütze oder Übungen am Reck, gingen von der Annahme aus, dass Mädchen körperlich nicht in der Lage wären, diese auszuführen.

Würden nur die Beschreibungen der Lernziele und die darin verankerten „Besonderheiten" für Mädchen, nicht aber die Senkung der Stundenzahlen in einigen Fächern einer genaueren Betrachtung unterzogen, könnte das Ergebnis lauten, dass Mädchen durch das „Reichsvolksschulgesetz" zusätzlich zur allgemeinen Ausbildung der Knaben noch gezielten, auf ihre Bedürfnisse abgestimmten Unterricht erhielten. Von all diesen Bestimmungen waren jedoch vor allem Mädchen im städtischen Bereich betroffen, da am Lande Volksschulen mit vier oder mehr Klassen nur sehr selten zu finden waren. ${ }^{90}$

\subsubsection{Mädchenvolksschulen}

Die Trennung der Geschlechter wurde durch das „Reichsvolksschulgesetz“ nicht explizit gefordert, war aber erwünscht. Wann eigenständige Mädchenschulen errichtet werden sollten, wurde der Landesgesetzgebung überlassen. Doch auch in den meisten Gesetzblättern der Länder findet man keine genauen Angaben dazu, wann Mädchenschulen einzurichten waren. In Salzburg und der Steiermark hieß es zu dieser Thematik etwa, dass die gemischten Volksschulen getrennt und eigene Mädchenschulen errichtet werden sollten , ,soweit es die vorhandenen Mittel gestatten.“91 Verpflichtend waren die geschlechtsspezifische Trennung und die Errichtung einer Mädchenschule auf jeden Fall, wenn die allgemeine Volksschule in einem Ort mehr als sechs Klassen hatte. ${ }^{92}$

Es war durchaus erlaubt, dass an reinen Mädchenschulen männliche Lehrkräfte unterrichteten. Allerdings musste, wenn sonst nur männliche Lehrpersonen an der Schule waren, eine weibliche Lehrerin für den Unterricht in "Weiblichen Handarbeiten" herangezogen werden. $\mathrm{Ob}$ andersherum Knaben auch von weiblichen Lehrkräften unterrichtet werden durften, sollte von der Landesgesetzgebung bestimmt werden. In Salzburg und der Steiermark durften Jungen beispielsweise nur in den unteren vier Jahrgängen von Frauen unterrichtet werden, auch in gemischten Schulen, in denen neben den Knaben auch Mädchen in den Klassen waren..$^{93}$

Wurden Mädchen und Jungen getrennt voneinander unterrichtet, lässt sich feststellen, dass die Erziehung der Mädchen meist stärker katholisch geprägt war als die der

90 Friedrich, Ein Paradies ist uns verschlossen...., S. 239.

91 Gemäß §4 Gesetz zur Regelung der Errichtung, der Erhaltung und des Besuchs der öffentlichen Volksschulen, Landesgesetz- und Verordnungsblatt für das Herzogtum Steiermark i. d. F., 8. März 1870, [http://alex.onb.ac.at/ cgi-content/alex?aid=Igm\&amp;datum=1870\&amp;page=29\&amp;size=45], eingesehen 14.3.2018. 
Knaben. Das lag daran, dass staatliche Stellen nach dem "Reichsvolksschulgesetz" schnell dazu übergingen, privaten, meist von Orden geführten Mädchenvolksschulen das Öffentlichkeitsrecht zu verleihen, um keine neuen staatlich finanzierten Schulen errichten zu müssen. ${ }^{94}$ In Tirol lag die Mädchenbildung um 1900 zum größten Teil noch in den Händen von katholischen Schulorden. ${ }^{95}$

\subsection{Geschlechtsspezifische Unterschiede in Bürgerschulen}

Die Bedeutung der Bürgerschule für die Mädchenbildung ist nicht zu unterschätzen. Immerhin stellte der Abschluss der Bürgerschule bis zur Ersten Republik den höchsten allgemeinbildenden Schulabschluss dar, den ein Mädchen erlangen konnte. ${ }^{96} \mathrm{Im}$ Gegensatz zur Volksschule war die Trennung der Geschlechter in der Bürgerschule nicht nur erwünscht, sondern gesetzlich vorgeschrieben. Diese Bestimmung wurde auch in der Praxis eingehalten. Im Schuljahr 1870/71 waren nur vier von insgesamt 43 Bürgerschulen in Österreich für beide Geschlechter zugänglich. Die Trennung der Geschlechter war schon allein aus dem Grund notwendig, dass die Bürgerschule für Mädchen und Buben zwei unterschiedliche Bildungsziele verfolgte. Während sie für die Knaben die Möglichkeit für den Besuch einer weiterführenden Schule bot, sollte sie die Mädchen dazu befähigen, einem Haushalt vorzustehen. ${ }^{97}$ Diesem Bildungsziel entsprechend wich auch der Lehrplan für Mädchenbürgerschulen von dem der Knaben ab. So erhielten Mädchen weniger Unterricht in den Fächern „Unterrichtssprache”, "Naturlehre“, „Arithmetik", "Geometrische Formenlehre“, "Freihandzeichnen“ und „Schreiben". Ab der Novellierung 1883 war der Turnunterricht für Mädchen nicht mehr obligat. Wie in den anderen Grundschulformen erhielten Schülerinnen in der Bürgerschule zusätzlichen Unterricht in „Weiblichen Handarbeiten“98 Die Lehrerin und österreichische Frauenrechtlerin Auguste Fickert sah diese Entwicklung sehr kritisch. Ihrer Meinung nach erlitten Mädchen

„insofern eine Beeinträchtigung, als für sie das Stundenausmass für den Unterricht in Sprache und in Rechnen reduziert und der Turnunterricht als nicht obligat erklärt wurde, wodurch in der Bevölkerung das Vorurteil bestärkt wird, dass diese Disziplin als, unweiblich' zu verwerten sei, dass man besser daran thue, diese Zeit mit Handarbeit auszufüllen."99

\footnotetext{
94 Dieses System wurde auch auf Mädchenbürgerschulen angewandt; Friedrich, Stieftöchter, S. 307.

95 Fickert, Der Stand der Frauenbildung in Österreich, S. 164.

96 Margret Friedrich, "Die Schülerinnen werden liebevoll behandelt - im Ganzen herrscht Zucht und Ordnung”. Die Tätigkeit der weiblichen Schulorden in Salzburg, in: Ilse Brehmer/Gertrud Simon (Hrsg.), Geschichte der Frauenbildung und Mädchenerziehung in Österreich. Ein Überblick, Graz 1997, S.108-127, hier S. 115.

97 Fischer-Kowalski u. a., Von den Tugenden der Weiblichkeit, S. 46; Siegmund Kraus, Zur Bürgerschulreform, in: Neues Frauenleben XV (April 1903), S.1-2; Margret Friedrich, „Ein Paradies ist uns verschlossen...." Zur Geschichte der schulischen Mädchenerziehung in Österreich im „Iangen“ 19. Jahrhundert, Wien-Köln-Weimar 1999, S. 63. Lehrplan der achtclassigen Bürgerschule für Mädchen. Veröffentlicht durch Verordnung des k. k. Unterrichtsministers vom 18. Mai 1874. Z 6549, Wien 1874, S. 2. 
Dennoch ist hier zu erwähnen, dass dieser Unterricht wesentlich zur Autonomie junger Frauen beitrug.

Trotz des teilweise sehr großen Unterschiedes in der Anzahl der Wochenstunden galten für die einzelnen Unterrichtsgegenstände in Mädchenbürgerschulen - zumindest in der Theorie - die gleichen Lehrziele wie in Knabenbürgerschulen. ${ }^{100}$

Schon wenige Jahre nach der Durchführung des "Reichsvolksschulgesetzes" wurde festgestellt, dass nur wenige Mädchen die Möglichkeit nutzten, die Bürgerschule drei Jahre lang zu besuchen. Außerdem waren die Schulversäumnisse in Mädchenbürgerschulen um einiges höher als in denen für Knaben. 1899 wurde überlegt, in Volksschulen, die mit einer Bürgerschule kombiniert waren, eine Abschlussklasse für all diejenigen einzuführen, die nach Beendigung der Volksschule die Bürgerschule nicht besuchen konnten oder wollten. Von Lehrerinnenvereinen wurde jedoch befürchtet, dass damit noch weniger Mädchen in die Bürgerschule übertreten würden, da das geplante Abschlusszeugnis sie zur Arbeit als Dienstmädchen oder zur Mithilfe im elterlichen Haushalt bemächtigen würde. Außerdem wurde davon ausgegangen, dass auch für weibliche Lehrlinge kein höheres Zeugnis mehr gefordert werden würde. Ein höherer Schulabschluss wäre somit für Mädchen nicht mehr notwendig, während von Knaben mindestens ein Abschlusszeugnis der zweiten Klasse der Bürgerschule, meistens sogar der dritten Klasse, verlangt wurde. ${ }^{101}$

1903 wurde die Bürgerschule um ein viertes freiwilliges Schuljahr erweitert. Für Jungen bedeutete der Abschluss dieses zusätzlichen Jahres die Gleichstellung mit einem Mittelschulabschluss. Für Mädchen war diese Gleichstellung allerdings nicht vorgesehen. Zwar hatten sie auch die Möglichkeit, die Bürgerschule ein weiteres Jahr lang zu besuchen, jedoch sollte sie dieser einjährige Lehrgang für das praktische Leben ausbilden. Sie erlernten dort nur eine zweite lebende Fremdsprache, "Stenographie" sowie "die nötigen Kenntnisse zur Führung eines Haushaltes, [...] Hygiene und Krankenpflege."102 Die Möglichkeit, die Bürgerschule um ein weiteres Schuljahr zu verlängern, wurde nur von wenigen Mädchen wahrgenommen. Allgemein besuchten Mädchen die Bürgerschule nur selten bis zum letzten Jahr. Die meisten Mädchen - besonders aus ärmeren Schichten - mussten die Schule bereits im Alter von zwölf Jahren verlassen, um im elterlichen Haushalt mitzuhelfen oder um Geld zu verdienen. Doch auch für Mädchen aus wohlhabenden Familien stellte der Besuch aller drei beziehungsweise vier Bürgerschuljahre ein Privileg dar. So traten beispielsweise 52 Mädchen im Schuljahr 1897 in die Bürgerschule der Vöcklabrucker Schulschwestern in der Stadt Salzburg ein. Nur zwanzig von ihnen besuchten die Schule für die gesamten drei Jahre. Dies ist umso bemerkenswerter, wenn man beachtet, dass allgemein nur etwa halb so viele Mädchen wie Jungen in die Bürgerschule eintraten. ${ }^{103}$

100 Lehrplan Mädchenbürgerschule, S. 1-14.

101 Friedrich, „Ein Paradies ist uns verschlossen...., S. 64-65.

102 Ebd., S. 63

103 Ebd., S. 65; Fischer-Kowlaski u. a., Von den Tugenden der Weiblichkeit, S. 19. 
Auf der anderen Seite sahen sich Mädchen, die die Lehrerinnenbildungsanstalt besuchen wollten, gezwungen, die letzte Klasse der Bürgerschule zu wiederholen, da die Lehrerbildungsanstalten Schüler_innen erst ab einem Alter von 15 Jahren aufnahmen, die Mädchen die Bürgerschule im Regelfall aber schon mit 14 Jahren abschlossen. Fortbildungskurse, die auf die Aufnahmeprüfung an den Lehrerbildungsanstalten vorbereiteten oder andere Möglichkeiten, die Zeit zwischen Abschluss der Bürgerschule und frühester Aufnahme an den Anstalten zu überbrücken, gab es nicht. ${ }^{104}$

„So wiederholen die Absolventinnen der Bürgerschulen, welche sich dem Lehrberuf widmen wollen, die letzte Klasse und zwar oft 2 bis 3 mal, da der Andrang zu der Aufnahmsprüfung die aufzunehmende Zahl der Kandidatinnen oft um das 5 fache übersteigt." ${ }^{105}$

Obwohl sie im Gesetz noch immer verankert war, wurde die Geschlechtertrennung in den Bürgerschulen zu Beginn des zwanzigsten Jahrhunderts in der Praxis teilweise nicht mehr eingehalten. Beispielsweise war es in Preßnitz (Př́sečnice) ${ }^{106}$ durch einen ministerialen Erlass erlaubt, dass Mädchen und Knaben die vierte Klasse der Bürgerschule gemeinsam besuchten. Kurz darauf wurde allgemein erlaubt, dass Mädchen in Orten, in denen es keine eigene Mädchenbürgerschule gab, die Knabenbürgerschule besuchten. ${ }^{107}$

\section{Fazit}

Am Beginn dieser Arbeit wurde die These aufgestellt, dass das „Reichsvolksschulgesetz" zwar eine wichtige Innovation für das österreichische Grundschulwesen war, die Bildungschancen für Mädchen sich dadurch allerdings nur unwesentlich verbesserten.

Ohne Zweifel lässt sich feststellen, dass das „Reichsvolksschulgesetz" den Höhepunkt der Veränderungen des österreichischen Grundschulwesens im 19. Jahrhundert darstellte. Der erste Schritt zu dieser Reform war die Erkenntnis, dass die bisherige Elementarbildung nicht mehr dem Stand der Zeit entsprach und die Schüler_innen im Vergleich zu anderen Ländern nicht ausreichend ausgebildet wurden. Von der Erweiterung der Unterrichtsgegenstände über die Einführung einheitlicher Lehrpläne bis zur Verlängerung der Schulpflicht brachte das „Reichsvolksschulgesetz" zahlreiche Änderungen mit sich.

Da das „Reichsvolksschulgesetz" einen enormen Umschwung bedeutete, wurde es von vielen Seiten kritisiert. Trotz aller Kritik muss jedoch festgehalten werden, dass viele der Neuerungen, die das „Reichsvolksschulgesetz" mit sich brachte, innovative Ideen beinhalteten, die das österreichische Grundschulwesen für lange Zeit stark beeinflussten. ${ }^{108}$

\footnotetext{
104 Friedrich, Ein Paradies ist uns verschlossen...," S. 65.

105 Fickert, Der Stand der Frauenbildung in Österreich, S. 170-171.

106 Stadt in Tschechien, die mittlerweile von einem Stausee bedeckt ist.

107 Friedrich, „Ein Paradies ist uns verschlossen..., S. 64; O. A., Die Erfolge der Koedukation an den Bürgerschulen, in: Salzburger Volksblatt, Nr. 16, 20.1.1911, S. 2-3, [http://anno.onb.ac.at/cgi-content/anno?aid=svb\&amp;datum=19 110120\&amp;seite=2\&amp;zoom=33], eingesehen 13.3.2018.

108 Dafür spricht auch, dass das „Reichsvolksschulgesetz“ beinahe 100 Jahre in Kraft war.
} 
Über die Frage, ob das "Reichsvolksschulgesetz" die Bildungssituation der beiden Geschlechter gleichermaßen verbesserte, lässt sich streiten. Auch wenn Unterrichtsminister Leopold Hasner von Artha bereits vor dem Inkrafttreten des „Reichsvolksschulgesetzes" erkannt hatte, dass Mädchen mehr Bildung benötigten, lässt sich doch in Frage stellen, ob dies mit dem „Reichsvolksschulgesetz" gelungen ist. Klar festzustellen ist jedoch, dass eine Gleichstellung der Geschlechter nicht das Ziel des „Reichsvolksschulgesetzes" war. Weder die Volks- noch die Bürgerschulen sahen die gleichen Bildungsziele für Mädchen und Knaben vor. Die gesellschaftlichen Rollenbilder ließen für Mädchen nur eine auf ihre „speziellen Bedürfnisse“ angepasste Ausbildung zu. Die Theorie der "Natur der Frau", die sich klar von der des Mannes unterschied, war fest in der Gesellschaft verankert und so war an eine gleichberechtigte Koedukation der Geschlechter noch nicht zu denken.

Zusammengefasst lässt sich festhalten, dass das "Reichsvolksschulgesetz" ein wichtiger Schritt in der Reformierung des österreichischen Grundschulwesens war, welcher jedoch nicht primär auf die Verbesserung der Situation der Mädchenausbildung abzielte.

\section{Literatur}

Boyer, Ludwig, Elementarschulen und Elementarunterricht in Österreich. Illustrierte Chronik der Schul- und Methodengeschichte von den ältesten Quellen bis zum Ende des 19. Jahrhunderts, Graz 2012.

Burow, Julie, Ueber die Erziehung des weiblichen Geschlechts, 1856, in: Günter Häntschel (Hrsg.), Bildung und Kultur bürgerlicher Frauen 1850-1918. Eine Quellendokumentation aus Anstandsbüchern und Lebenshilfen für Mädchen und Frauen als Beitrag zur weiblichen literarischen Sozialisation, Tübingen 1986, S. 88-107.

Engelbrecht, Helmut, Geschichte des österreichischen Bildungswesens. Erziehung und Unterricht auf dem Boden Österreichs. Von der frühen Aufklärung bis zum Vormärz, Bd. 3, Wien 1984.

Engelbrecht, Helmut, Geschichte des österreichischen Bildungswesens. Erziehung und Unterricht auf dem Boden Österreichs. Von 1848 bis zum Ende der Monarchie, Bd. 4, Wien 1986.

Fickert, Auguste, Handbuch der Frauenbewegungen. Der Stand der Frauenbildung in Österreich, in: Gertrud Bäumer/Helene Lange (Hrsg.), Der Stand der Frauenbildung in den Kulturländern, Berlin 1902, S. 160-190.

Fischer-Kowalski, Maria u. a., Von den Tugenden der Weiblichkeit. Mädchen und Frauen im österreichischen Bildungssystem, Wien 1986.

Friedrich, Margret, „Die Schülerinnen werden liebevoll behandelt - im Ganzen herrscht Zucht und Ordnung". Die Tätigkeit der weiblichen Schulordnung in Salzburg, in: Ilse Brehmer/Gertrud Simon (Hrsg.), Geschichte der Frauenbildung und Mädchenerziehung in Österreich. Ein Überblick, Graz 1997, S. 108-127. 
Friedrich, Margret, Ein Paradies ist uns verschlossen...." Zur Geschichte der schulischen Mädchenerziehung in Österreich im „langen“ 19. Jahrhundert, Wien-Köln-Weimar 1999.

Friedrich, Margret, Hatte Vater Staat nur Stieftöchter? Die Maßnahmen des österreichischen Unterrichtsministeriums zur Mädchenbildung 1848-1919, in: Brigitte Mazohl (Hrsg.), Bürgerliche Frauenkultur im 19. Jahrhundert (L'homme Schriften 2), Wien 1995, S. 301-342.

Haselwanter, Franz, Die Entwicklung des Volks- und Bürgerschulwesens. In den Jahren 1910 bis 1927, Innsbruck 1985.

Vocelka, Karl, Verfassung oder Konkordat? Der publizistische und politische Kampf der österreichischen Liberalen um die Religionsgesetze des Jahres 1868 (Studien zur Geschichte der österreichisch-ungarischen Monarchie XVII), Wien 1978.

\section{Quellen (chronologisch geordnet)}

Allgemeine Schulordnung für die deutschen Normal- Haupt- und Trivialschulen in sämmtlichen kaiserl. königl. Erbländern d.d. Wien den 6ten December 1774, [https://www.digital.wienbibliothek.at/wbrobv/content/titleinfo/1843007], eingesehen 14.3.2018.

Politische Verfassung der deutschen Schule in der k. auch k.k. deutschen Erbstaaten, Wien 1806, [https://books.google.at/books?id=IB9OAAAAcAAJ\&printsec=frontcover\& $\mathrm{hl}=\mathrm{de} \# \mathrm{v}=$ onepage\&q\&f=false], eingesehen 14.3.2018.

Gesetz vom 25. Mai 1868 wodurch grundsätzliche Bestimmungen über das Verhältniß der Schule zur Kirche erlassen werden, RGBI. Nr. 48, [http://alex.onb.ac.at/cgi-content/ alex?aid=rgb\&datum $=1868 \&$ size $=45 \&$ page $=125]$, eingesehen 9.3.2018.

Gesetz vom 14. Mai 1869, durch welches die Grundsätze des Unterrichtswesens bezüglich der Volksschule festgelegt werden, RGBI. Nr. 62, [http://alex.onb.ac.at/cgi-content/ alex?aid=rgb\&datum=18690004\&seite=00000277], eingesehen 9.3.2018.

Verordnung des Ministers für Cultus und Unterricht vom 12. Juli 1869, womit Uebergangsbestimmungen zur Durchführung des Volksschulgesetzes erlassen werden, RGBI. Nr. 130, [http://alex.onb.ac.at/cgi-content/alex?aid=rgb\&datum $=1869 \&$ page $=48$ $6 \&$ size=45], eingesehen 9.3.2018.

Gesetz zur Regelung der Errichtung, der Erhaltung und des Besuchs der öffentlichen Volksschulen, Landesgesetz- und Verordnungsblatt für das Herzogtum Steiermark, 8. März 1870, [http://alex.onb.ac.at/cgi-content/alex?aid=lgm\&datum=1870\&page=29\& size=45], eingesehen 14.3.2018.

Verordnung des Ministers für Unterricht und Cultus vom 20. August 1870, womit eine Schul- und Unterrichtsordnung für die allgemeinen Volksschulen erlassen wird, RGBI. Nr. 105, [http://alex.onb.ac.at/cgi-content/alex?aid=rgb\&datum $=18700004 \&$ sei te=00000219], eingesehen 14.3.2018. 
Gesetz vom 2. Mai 1883, womit einige Bestimmungen des Gesetzes vom 14. Mai 1869 (RGBI. Nr.62), abgeändert werden, RGBI Nr. 53, [http://alex.onb.ac.at/cgi-content/alex?a $i d=r g b \&$ datum $=1883 \&$ size $=45 \&$ page $=225$ ], eingesehen 12.3.2018.

Lehrplan für ungetheilte einclassige Volksschulen (mit ganztägigem Unterrichte). Veröffentlicht durch Verordnung des k. k. Unterrichtsministers vom 18. Mai 1874. Z. 6549, Wien 1874.

Lehrplan für zweiclassige Volksschulen. Veröffentlicht durch Verordnung des k. k. Unterrichtsministers vom 18. Mai 1874. Z. 6549, Wien 1874.

Lehrplan für siebenclassige Volksschulen. Veröffentlicht durch Verordnung des k. k. Unterrichtsministers vom 18. Mai 1874. Z 6549, Wien 1874.

Lehrplan der achtclassigen Bürgerschule für Mädchen. Veröffentlicht durch Verordnung des k. k. Unterrichtsministers vom 18. Mai 1874. Z 6549, Wien 1874.

O. A., Die Erfolge der Koedukation an den Bürgerschulen, in: Salzburger Volksblatt, Nr. 16, 20.1.1911, [http://anno.onb.ac.at/cgi-content/anno?aid=svb\&amp;datum=191101 20\&amp;seite=2\&amp;zoom=33], eingesehen 13.3.2018.

Nina Hechenblaikner ist Studentin der Geschichtswissenschaften und studentische Mitarbeiterin am Institut für Zeitgeschichte an der Universität Innsbruck. Nina. Hechenblaikner@student.uibk.ac.at

\section{Zitation dieses Beitrages}

Nina Hechenblaikner, Das „Reichsvolksschulgesetz" und seine Auswirkungen auf die Mädchenbildung. Ein Schritt zur Gleichstellung der Geschlechter in österreichischen Grundschulen?, in: historia.scribere 10 (2018), S. 241-265, [http://historia.scribere.at], eingesehen 19.6.2018 (=aktuelles Datum).

Creative Commons Licences 3.0 Österreich unter Wahrung der Urheberrechte der Autorlnnen. 
